\title{
Outcomes of the Different Types of Total Knee Arthroplasty with the Identical Femoral Geometry
}

\author{
Sang Min Lee, $\mathrm{MD}^{1}$, Sang Cheol Seong, $\mathrm{MD}, \mathrm{PhD}^{2}$, Sahnghoon Lee, $\mathrm{MD}, \mathrm{PhD}^{2}$, Won Chul Choi, $\mathrm{MD}^{3}$ and \\ Myung Chul Lee, $\mathrm{MD}, \mathrm{PhD}^{2}$ \\ ${ }^{1}$ Department of Orthopedic Surgery, Eulji University College of Medicine, Daejeon; ${ }^{2}$ Department of Orthopedic Surgery, Seoul National University College of Medicine, \\ Seoul; ${ }^{3}$ Department of Orthopedic Surgery, CHA Bundang Medical Center, CHA University, Seongnam, Korea
}

\begin{abstract}
Purpose: There are controversies around the role of the posterior cruciate ligament and the effect of design modifications for high flexion in total knee arthroplasty (TKA). So, we compared the clinical outcomes of the cruciate retaining (CR), posterior stabilized (PS), and high flexion posterior stabilized (F-PS) designs in TKA with identical femoral geometry.

Materials and Methods: One hundred seventy nine knees with 3 different types of prostheses after a minimum 5-year follow-up were enrolled in this retrospective study: 45 with CR, 40 with PS and 94 with F-PS. The mean ages of these groups were 65.7, 67.2, and 67.5, and the mean durations of follow-up were 8.1, 8.0, and 6.8 years, respectively. We compared the range of motion, functional outcomes, and radiographic measurements at the 2-year follow-up and last follow-up.

Results: The maximal flexion angle was significantly lower in the CR group than the F-PS group at the 2-year follow-up. However, there was no significant difference at the last follow-up. Functional outcomes and survival rate of the three groups were similar at the last follow-up.

Conclusions: Three different types of TKAs (CR, PS and F-PS) with identical femoral geometry showed similar mid-term outcomes with regard to the range of motion, functional outcomes and survival rate.
\end{abstract}

Key words: Knee, Total knee arthroplasty, Posterior cruciate-retaining, Posterior cruciate-substituting, High-flex implant.

\section{Introduction}

Total knee arthroplasty (TKA) is a successful procedure for pain relief and functional restoration in patients with advanced osteoarthritis. Despite its success, a significant portion of patients are dissatisfied with their replaced knees because of their higher expectations on the function, such as high flexion activities ${ }^{1-3)}$. Therefore, implant manufacturers have introduced many

Received April 1, 2012; Revised (1st) May 7, 2012; (2nd) August 1, 2012; Accepted August 17, 2012.

Correspondence to: Myung Chul Lee, MD.

Department of Orthopedic Surgery, Seoul National University College of Medicine, 101 Daehang-ro, Jongno-gu, Seoul 110-744, Korea.

Tel: +82-2-2072-3212, Fax: +82-2-764-2718

Email: leemc@snu.ac.kr

This is an Open Access article distributed under the terms of the Creative Commons Attribution Non-Commercial License (http://creativecommons.org/licenses/by-nc/3.0/) which permits unrestricted non-commercial use, distribution, and reproduction in any medium, provided the original work is properly cited. modifications to the prosthesis design for a better outcome. Nevertheless, some of these changes, including the handling of the posterior cruciate ligament and the design modifications for high flexion, are still controversial around the clinical outcomes and longevity ${ }^{4-8)}$.

Surgeons with a preference for cruciate retaining (CR) knees set a high value on the function of the posterior cruciate ligament (PCL). They believe that the PCL has a beneficial effect on femoral rollback, quadriceps efficiency, joint stability and proprioception ${ }^{9,10)}$. On the other hand, other surgeons who favor posterior stabilized (PS) knees have doubts about the function of the remaining PCL in CR knees ${ }^{11}$. Recently, increasing attention has been on whether the PS knee is superior to CR knee in the range of motion ${ }^{4,5)}$.

Since the late 1990s, many implant manufacturers have made modifications to conventional prostheses designs to improve the maximal knee flexion. The design changes for high flexion had been in the polyethylene insert geometry, posterior femoral condylar offset, cam/post engagement, or a combination of these ${ }^{12-15)}$. Nevertheless, it is unclear whether these design modifications improve the range of motion and 
clinical outcomes $^{6-8,14,16)}$. The high flexion implants used in the current study has a relaxed posterior slope of the polyethylene insert, compared to the conventional PS implant. This change in polyethylene insert geometry results in a reduced femoral rise, improved collateral ligament function in deep flexion, and reduced torsional constraint that requires less torque for rotation ${ }^{12}$. Although a high flexion design is theoretically superior to its conventional design, there is a controversy about whether a high flexion design facilitates greater knee flexion ${ }^{12,15}$. In addition, there are still concerns regarding whether this change of polyethylene insert affects the knee functions or longevity of replaced knees.

The purpose of this study was to compare the clinical outcomes of CR, PS, and high flexion PS prostheses having identical femoral component geometry. We hypothesized that retaining or substituting PCL and the change of polyethylene insert geometry for high flexion did not make a difference to the clinical outcomes of TKA.

\section{Materials and Methods}

This study was approved by our institutional review board. The data of 164 patients who underwent 225 primary total knee arthroplasties for primary osteoarthritis using the Scorpio total knee system (Stryker Orthopaedics, Mahwah, NJ, USA) between January 1999, and December 2004, were reviewed retrospectively: 57 knees with the Scorpio CR implants, 50 knees with the Scorpio PS implants, and 118 knees with the Scorpio Flex PS (F-PS) implants (Fig. 1). We included only primary osteoarthritic knees and there was no post-traumatic osteoarthritic knees, rheumatoid knees, stiff knees (range of knee motion, $<60^{\circ}$ ) and severely deformed knees (tibiofemoral angle, $>$ varus $20^{\circ}$ ). Implant selection was chronologically done in order of the prostheses development and launching. Twenty eight patients (37 knees) dropped out due to follow-up loss or death before the minimum follow-up of 5 years. In addition, four patients ( 5 knees) were excluded because of concurrent disease that could seriously affect the knee function such as an intracerebral hemorrhage, severe cerebral infarct and severe dementia. Another four knees were revised due to aseptic loosening or prosthesis infection before the 5-year follow-up. Aseptic loosening was observed in the PS implant (1 knee) and F-PS implant ( $2 \mathrm{knee})$. A prosthesis infection occurred in the CR implant. However, we did not exclude these four knees in the survival analysis. Of the remaining 179 knees (131 patients), CR implants were used in 45 knees (CR group), PS implants were used in 40 knees (PS group) and F-PS implants were used in 94 knees (F-PS group). The mean follow-up duration was 7.4 years (median, 7 years; range, 5 to 12 years). The CR, PS, and F-PS groups were followed-up for a mean of 8.1 years (range, 5 to 9.7 years), 8.0 years (range, 5 to 12 years) and 6.8 years (range, 5 to 8.8 years), respectively. Thirty six patients had bilateral total knee arthroplasties with the same prosthesis (6 patients with bilateral CR knees, 6 patients with bilateral PS knees and 24 patients with bilateral F-PS knees). Twelve patients underwent bilateral arthroplasty in which a different type of prosthesis was implanted in each knee (CR and PS, 3 patients; PS and F-PS, 2 patients; CR and F-PS, 7 patients).

All surgical procedures were performed by two experienced surgeons. Identical surgical techniques were used in the three groups, except for preservation of the PCL and larger posterior tibial slope in the CR group. An attempt was made to produce tibial slope of $6^{\circ}$ in the CR knees, and $3^{\circ}$ in the PS and F-PS knees. A medial parapatellar arthrotomy was used, and the patellae were routinely resurfaced. The patellae with normal cartilage or small patellae (less than or equal to $20 \mathrm{~mm}$ in thickness) were preserved. All prostheses were fixed with cement. The same rehabilitation protocol was applied to all groups in postoperation. Patients began knee flexion exercises using a continuous passive motion exercise machine on the first day after the surgery and
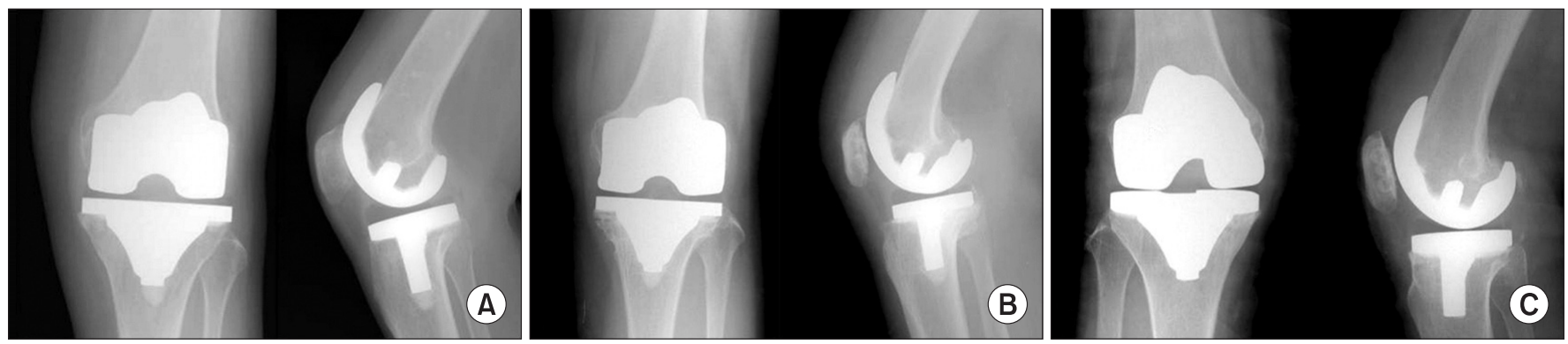

Fig. 1. Anterior/posterior and lateral radiographs of the knees with (A) cruciate retaining, (B) posterior stabilized and (C) high flexion posterior stabilized prostheses. 
were allowed to walk using a walker on the second day.

Clinical and radiographic evaluations were performed three months and one year after surgery and annually thereafter. However, postoperative knee scores have been regularly assessed since 2005. The active maximal knee-flexion angle and flexion contracture were measured using a goniometer with a patient in the supine position. Each knee was rated in accordance with the Knee Society and the Hospital for Special Surgery (HSS) scoring systems. In addition, each patient completed the Western Ontario and McMaster Universities Osteoarthritis Index (WOMAC) health status questionnaire. The radiographic indices were measured on standard weight-bearing anteroposterior and long leg radiographs, supine lateral radiographs $\left(30^{\circ}\right.$ flexed $)$ and merchant radiographs. The tibiofemoral angle, posterior tibial slope, changes in the posterior condylar offset and joint line level were assessed. The joint line level was evaluated by the distance from the distal femoral condyle to the proximal fibular tip in the anteroposterior radiographs.

All statistical analyses were performed using two-tailed tests. A p-value of $<0.05$ was considered significant. The categorical variables were analyzed using a cross-tabulation with a chisquare. The continuous variables (i.e., age, body mass index [BMI], preoperative flexion angle, Knee Society scores) were examined with analysis of variance and Scheffe's post hoc test. An analysis of covariance was also performed to analyze the postoperative flexion angle with consideration of the preoperative flexion angle, age, BMI and preoperative tibiofemoral angle. The survival rate was estimated by Kaplan-Meier analysis using a revision for any reason as an end point. A log-rank test was used to test for the differences in the survival curve by an implant subtype.

\section{Results}

There was no significant difference between the three groups in terms of the demographics and preoperative measurements except for the follow-up duration (Table 1).

At the 2-year follow-up, the mean active maximal flexion angles in the CR, PS, and F-PS groups were $114^{\circ}, 119^{\circ}$, and $122^{\circ}$, respectively. The maximal flexion was significantly lower in the CR group than the F-PS group $(\mathrm{p}=0.013)$. On the other hand, the differences in the maximal flexion angle between the three groups decreased with time. Eventually, there was no significant difference in the active maximal flexion at the last follow-up. After adjusting for age, BMI and preoperative tibiofemoral angle, the mean maximal flexion angles of the CR knees was also significantly lower than that of the F-PS knees at the 2-year follow-up $(\mathrm{p}<0.001)$, and no significant difference was observed at the last follow-up (Table 2).

The average postoperative Knee Society knee score, Knee

Table 1. Preoperative Demographics and Clinical Status of the Patients

\begin{tabular}{|c|c|c|c|c|}
\hline & CR group & PS group & F-PS group & p-value \\
\hline Total no. of knees & 45 & 40 & 94 & \\
\hline Gender $(\mathrm{M} / \mathrm{F})$ & $2 / 43$ & $3 / 37$ & $7 / 87$ & $0.613^{\mathrm{a}}$ \\
\hline Mean age (yr) & 65.7 & 67.2 & 67.5 & $0.352^{\mathrm{b}}$ \\
\hline Mean duration of follow-up (yr) & 8.1 & 8.0 & 6.8 & $<0.001^{\mathrm{b}}$ \\
\hline Patella (resurfaced/retained) & $41 / 4$ & $38 / 2$ & $80 / 14$ & $0.212^{\mathrm{a}}$ \\
\hline KS-Knee score (points) & $40 \pm 17.8$ & $38 \pm 17.3$ & $40 \pm 17.3$ & $0.858^{\mathrm{b}}$ \\
\hline KS-Function score (points) & $42 \pm 15.4$ & $42 \pm 14.3$ & $42 \pm 19.6$ & $0.995^{\mathrm{b}}$ \\
\hline HSS score (points) & $42 \pm 9.8$ & $44 \pm 10.0$ & $44 \pm 9.7$ & $0.600^{\mathrm{b}}$ \\
\hline WOMAC total score (points) & $59 \pm 15.4$ & $53 \pm 16.1$ & $56 \pm 16.4$ & $0.344^{\mathrm{b}}$ \\
\hline Body mass index $\left(\mathrm{kg} / \mathrm{m}^{2}\right)$ & $27 \pm 2.5$ & $27 \pm 3.3$ & $28 \pm 4.2$ & $0.339^{\mathrm{b}}$ \\
\hline Tibiofemoral angle $\left({ }^{\circ}\right)$ & Varus $6 \pm 4.4$ & Varus $7 \pm 7.6$ & Varus $5 \pm 8.5$ & $0.154^{\mathrm{b}}$ \\
\hline Flexion contracture angle $\left({ }^{\circ}\right)$ & $13 \pm 6.8$ & $15 \pm 8.2$ & $14 \pm 10.2$ & $0.750^{\mathrm{b}}$ \\
\hline Active maximal flexion angle $\left({ }^{\circ}\right)$ & $126 \pm 11.6$ & $124 \pm 16.3$ & $124 \pm 16.8$ & $0.784^{\mathrm{b}}$ \\
\hline Total range of motion $\left({ }^{\circ}\right)$ & $112 \pm 14.6$ & $109 \pm 20.7$ & $110 \pm 23.6$ & $0.701^{\mathrm{b}}$ \\
\hline
\end{tabular}

Values are presented as number or mean \pm standard deviation.

CR: cruciate retaining, PS: posterior stabilized, F-PS: flexion posterior stabilized, KS: Knee Society, HSS: Hospital for Special Surgery, WOMAC: Western Ontario and McMaster Universities Osteoarthritis Index.

${ }^{\text {a) }}$ Chi-square test, ${ }^{\text {b) }}$ Analysis of variance test. 
Table 2. Comparison of Postoperative Ranges of Knee Motion between the Groups

\begin{tabular}{|c|c|c|c|c|c|}
\hline & & CR group $(n=45)$ & PS group $(n=40)$ & F-PS group $(n=94)$ & $\mathrm{p}$-value \\
\hline \multirow[t]{3}{*}{2 years follow-up } & Flexion contracture ${ }^{\mathrm{a})}$ & $2 \pm 3.9$ & $1 \pm 2.9$ & $2 \pm 3.0$ & $0.395^{\mathrm{c})}$ \\
\hline & Active maximal flexion ${ }^{\text {a) }}$ & $114 \pm 14.2$ & $119 \pm 10.1$ & $122 \pm 15.8$ & $0.013^{(, e)}$ \\
\hline & Total range of motion ${ }^{a}$ & $113 \pm 16.8$ & $118 \pm 11.5$ & $120 \pm 17.0$ & $0.040^{\mathrm{c})}$ \\
\hline \multirow[t]{3}{*}{ Last follow-up } & Flexion contracture ${ }^{\text {a) }}$ & $1 \pm 4.1$ & $1 \pm 1.5$ & $2 \pm 4.6$ & $0.220^{c)}$ \\
\hline & Active maximal flexion ${ }^{\text {b) }}$ (adjusted) & $116 \pm 2.0$ & $121 \pm 2.0$ & $120 \pm 1.4$ & $0.173^{\mathrm{d})}$ \\
\hline & Total range of motion ${ }^{a}$ & $115 \pm 15.8$ & $121 \pm 11.9$ & $118 \pm 17.7$ & $0.319^{c)}$ \\
\hline
\end{tabular}

CR: cruciate retaining, PS: posterior stabilized, F-PS: flexion posterior stabilized.

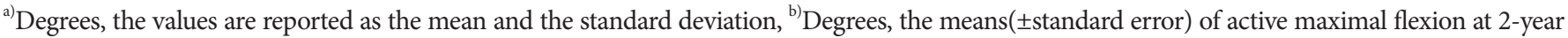
follow-up and last follow-up between the three groups adjusted by age, gender, body mass index, preoperative maximal flexion angle and preoperative tibiofemoral angle, ${ }^{\mathrm{c}}$ Analysis of variance test, ${ }^{\mathrm{d})}$ Analysis of covariance test, ${ }^{\mathrm{e})}$ Significance determined by analysis of variance or analysis of covariance with Scheffe's post hoc method; for active maximal flexion and total range of motion at 2-year follow-up, F-PS group was significantly higher than CR group.

Table 3. Comparison of Postoperative Knee Scores between the Groups

\begin{tabular}{lcccc}
\hline \multicolumn{1}{c}{ Last follow-up } & $\begin{array}{c}\text { CR group } \\
(\mathrm{n}=45)\end{array}$ & $\begin{array}{c}\text { PS group } \\
(\mathrm{n}=40)\end{array}$ & $\begin{array}{c}\text { F-PS group } \\
(\mathrm{n}=94)\end{array}$ & p-value ${ }^{\mathrm{a})}$ \\
\hline KS score (points) & & & & \\
$\quad$ Knee & $93 \pm 9.7$ & $94 \pm 5.6$ & $94 \pm 6.5$ & 0.863 \\
$\quad$ Function & $73 \pm 25.0$ & $80 \pm 19.5$ & $77 \pm 22.8$ & 0.348 \\
HSS score (points) & $88 \pm 7.2$ & $90 \pm 5.0$ & $89 \pm 6.9$ & 0.485 \\
WOMAC score (points) & & & & \\
$\quad$ Pain & $1 \pm 2.0$ & $1 \pm 1.2$ & $1 \pm 1.7$ & 0.805 \\
$\quad$ Stiffness & $1 \pm 0.8$ & $1 \pm 1.1$ & $1 \pm 1.2$ & 0.280 \\
$\quad$ Function & $18 \pm 12.3$ & $15 \pm 9.1$ & $15 \pm 9.2$ & 0.382 \\
\hline
\end{tabular}

Values are presented as mean \pm standard deviation.

KS: Knee Society, HSS: Hospital for Special Surgery, WOMAC: Western Ontario and McMaster Universities Osteoarthritis Index.

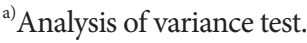

Society function score, HSS score, and WOMAC scores for the pain, stiffness and function subscales were similar between the groups at the last follow-up (Table 3).

Postoperative tibiofemoral angles averaged $6^{\circ}$ of valgus in the CR group, $6^{\circ}$ of valgus in the PS group, and $5^{\circ}$ of valgus in the F-PS group $(\mathrm{p}=0.376)$. The mean changes in the posterior femoral condylar offset were also similar between the three groups (CR, $1 \pm 1.9 \mathrm{~mm}$; PS, $1 \pm 2.3 \mathrm{~mm}$; F-PS, $1 \pm 2.2 \mathrm{~mm} ; \mathrm{p}=0.647$ ). The mean posterior tibial slope of the CR group was higher than the other groups (CR, $6^{\circ} \pm 1.8^{\circ}$; PS, $4^{\circ} \pm 2.8^{\circ}$; F-PS, $4^{\circ} \pm 2.3^{\circ} ; \mathrm{p}<0.001$ ). The joint line levels were $15 \mathrm{~mm}$ in the CR group, $14 \mathrm{~mm}$ in the PS group and $16 \mathrm{~mm}$ in the F-PS group ( $\mathrm{p}=0.201)$.

After a minimum follow-up of 5 years, two knees underwent

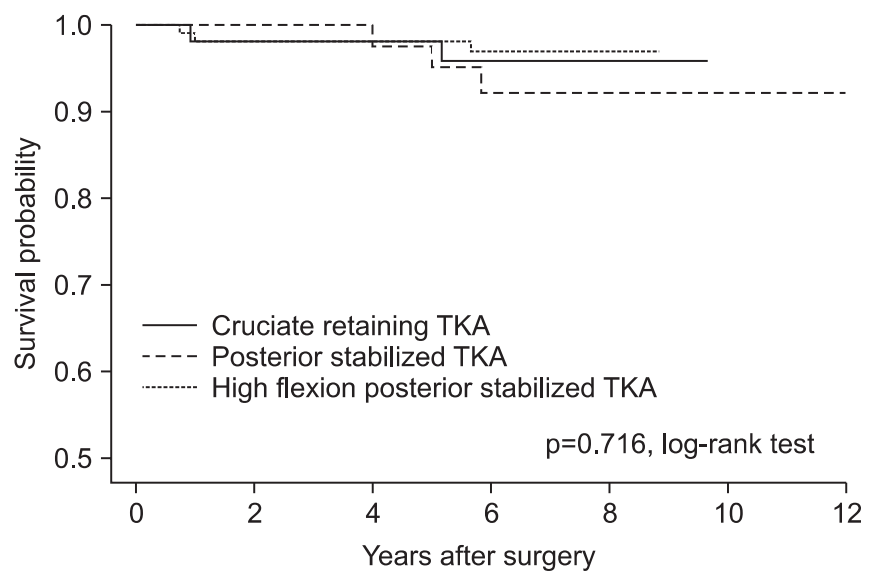

Fig. 2. Kaplan-Meier survivorship estimates, using revision surgery for any reason as an endpoint, showed a $96.1 \%$ survival rate at 7 years. The survival curves of the cruciate retaining, posterior stabilized, and high flexion posterior stabilized groups were similar. TKA, total knee arthroplasty.

revision surgery for aseptic loosening (CR, 1 knee; PS, 1 knee). Including the cases followed up for less than 5 years, aseptic loosening occurred in five knees (CR, 1 knees; PS, 2 knees; F-PS, 2 knees). Loosening only involved the tibial component, and the plates medially sank down in all cases. The mean time from TKA to revision for aseptic loosening was $3.2 \pm 2.15$ years. One PS knee was revised for periprosthetic fracture. There was no prosthesis infection after 5-year follow-up. The overall survival rate at 7 years was $96.1 \%$, and there was no significant difference in the survival curve between the three groups (CR, 95.9\%; PS, 92.3\%; F-PS, 97\%; $\mathrm{p}=0.716$ ) (Fig. 2). 


\section{Lee et al. Outcomes of the Different Types of Total Knee Arthroplasty}

\section{Discussion}

The role of the posterior cruciate ligament and the effect of the design modifications for high flexion are controversial subjects in TKA. This study compared the results of CR, PS, and F-PS prostheses with identical femoral component design.

In the current study, the CR group had a lower mean maximal flexion angle than the PS $\left(\Delta 5^{\circ}\right)$ or F-PS $\left(\Delta 9^{\circ}\right)$ group at the 2-year follow-up, even though there was only a significant difference between the CR and F-PS group. Many short-term studies also found that PS knees had significantly greater maximal flexion than CR knees ${ }^{4,17,18)}$. Although several studies showed that CR and PS knees had a similar range of motion, these studies had the mean preoperative maximal flexion of the CR knees at $4^{\circ}$ to $9^{\circ}$ higher than the PS knees ${ }^{9,19,20)}$.

In the present study, as the maximal flexion of the CR group increased, the difference in maximal flexion between the $\mathrm{CR}$ and F-PS groups decreased at the last follow-up. An increase in the maximal flexion at the mid-term follow-up was also observed in another CR study ${ }^{21}$, and a decrease in maximal flexion was observed in another PS study ${ }^{22}$. The change of flexion angle in the CR knee may be referable to a degeneration or failure of PCL. Victor et al. ${ }^{10)}$ reported that the mean flexion angle of the CR and PS designs were similar at the midterm follow-up. In contrast, Harato et al. ${ }^{5)}$ demonstrated significantly greater average knee flexion of the PS knees at 5 year follow-up. However, the difference in the mean flexion angle was only $3.3^{\circ}$.

In the current study, the mean flexion angle of the F-PS group was similar to that of the PS group. On the other hand, in the several randomized, controlled studies, high flexion PS designs have demonstrated an improved range of motion comparing to the conventional PS designs ${ }^{6,23,24)}$. In these studies, the design modifications for high flexion were mainly in the posterior femoral condylar offset and cam/post engagement. Other high flexion designs, which were characterized by changes in the polyethylene insert geometry without an increase of femoral condylar offset, did not show a significantly higher flexion arc than the conventional PS designs in the prospective study ${ }^{15}$.

Bellemans et al. ${ }^{25,26)}$ reported that the posterior femoral condylar offset and posterior tibial slope can affect the postoperative range of motion. In the current study, the femoral condylar offsets were similarly restored in the three groups, but the mean tibial slopes were significantly different. We intentionally attempted to make a smaller tibial slope in the PS and F-PS groups because an excessive slope in a PS knee can cause posterior flexion instability ${ }^{27}$. Bellemans et al. ${ }^{26)}$ stated that increasing the tibial slope improved maximal flexion, with an average of $1.7^{\circ}$ more flexion for every degree extra of the tibial slope. Therefore, the difference in maximal flexion between the CR and PS knees has the potential to be undervalued in the current study.

The survival rate in this study was $96.1 \%$ at 7 years, which is comparable to the other midterm studies using identical prostheses. Abbas and Gunn ${ }^{28)}$ reported a 99.3\% survival rate on 173 primary PS TKA at 8 years. Similarly, Borrione et al. ${ }^{29)}$ reported a 95.2\% survival rate at 6 years in the multicenter study. In contrast to Rand et al. ${ }^{30)}$, who reported a significantly lower survival rate for PS designs compared to CR designs, the survival curve of the CR group in the current study was similar to those of the other groups.

This study had several limitations. First, this study included a small population and was potentially underpowered, given a small difference in the clinical outcomes. Second, only twelve patients, who had a different type of prosthesis in each knee, were not included but both knees of the forty eight patients with bilateral arthroplasties were enrolled. This might have obscured the analysis. However, the functional status was assessed separately for each knee, and the outcomes were analyzed with consideration for possible confounding factors, such as preoperative flexion angle, age, BMI and preoperative tibiofemoral angle. Third, the index arthroplasties were carried out over a six year period, and the mean follow-up duration varied across the groups. Because the implants were chronologically chosen in order of the prosthesis development and launching, the mean follow-up durations were different between the groups.

\section{Conclusions}

In the present study, the retaining or substituting PCL and the change of polyethylene insert geometry for high flexion did not make a difference to the midterm clinical outcomes of TKA with identical femoral geometry. Although the maximal flexion was lower in the CR knee than the F-PS knee at 2-year followup, the difference in the maximal flexion decreased at the last follow-up. After a minimum follow-up of five years, the three different types of TKAs with identical femoral geometry provided similar outcomes with regard to the range of motion, functional outcomes and survival rate.

\section{Acknowledgements}

We thank the Medical Research Collaborating Center of 
Seoul National University Hospital for the help with statistical analysis. This study was supported by a grant from the Innovative Research Institute for Cell Therapy, Republic of Korea (Grant no. A062260).

\section{References}

1. Padua R, Ceccarelli E, Bondi R, Campi A, Padua L. Range of motion correlates with patient perception of TKA outcome. Clin Orthop Relat Res. 2007;460:174-7.

2. Argenson JN, Parratte S, Ashour A, Komistek RD, Scuderi GR. Patient-reported outcome correlates with knee function after a single-design mobile-bearing TKA. Clin Orthop Relat Res. 2008;466:2669-76.

3. Noble PC, Conditt MA, Cook KF, Mathis KB. The John Insall Award: Patient expectations affect satisfaction with total knee arthroplasty. Clin Orthop Relat Res. 2006;452:3543.

4. Maruyama S, Yoshiya S, Matsui N, Kuroda R, Kurosaka M. Functional comparison of posterior cruciate-retaining versus posterior stabilized total knee arthroplasty. J Arthroplasty. 2004;19:349-53.

5. Harato K, Bourne RB, Victor J, Snyder M, Hart J, Ries MD. Midterm comparison of posterior cruciate-retaining versussubstituting total knee arthroplasty using the Genesis II prosthesis. A multicenter prospective randomized clinical trial. Knee. 2008;15:217-21.

6. Weeden SH, Schmidt R. A randomized, prospective study of primary total knee components designed for increased flexion. J Arthroplasty. 2007;22:349-52.

7. Huang HT, Su JY, Wang GJ. The early results of high-flex total knee arthroplasty: a minimum of 2 years of follow-up. J Arthroplasty. 2005;20:674-9.

8. Nutton RW, van der Linden ML, Rowe PJ, Gaston P, Wade FA. A prospective randomised double-blind study of functional outcome and range of flexion following total knee replacement with the NexGen standard and high flexion components. J Bone Joint Surg Br. 2008;90:37-42.

9. Conditt MA, Noble PC, Bertolusso R, Woody J, Parsley BS. The PCL significantly affects the functional outcome of total knee arthroplasty. J Arthroplasty. 2004;19:107-12.

10. Victor J, Banks S, Bellemans J. Kinematics of posterior cruciate ligament-retaining and -substituting total knee arthroplasty: a prospective randomised outcome study. J Bone Joint Surg Br. 2005;87:646-55.

11. Fantozzi S, Catani F, Ensini A, Leardini A, Giannini S.
Femoral rollback of cruciate-retaining and posteriorstabilized total knee replacements: in vivo fluoroscopic analysis during activities of daily living. J Orthop Res. 2006;24:2222-9.

12. Klein GR, Parvizi J, Rapuri VR, Austin MS, Hozack WJ. The effect of tibial polyethylene insert design on range of motion: evaluation of in vivo knee kinematics by a computerized navigation system during total knee arthroplasty. J Arthroplasty. 2004;19:986-91.

13. Kim YH, Sohn KS, Kim JS. Range of motion of standard and high-flexion posterior stabilized total knee prostheses. A prospective, randomized study. J Bone Joint Surg Am. 2005;87:1470-5.

14. Choi WC, Lee S, Seong SC, Jung JH, Lee MC. Comparison between standard and high-flexion posterior-stabilized rotating-platform mobile-bearing total knee arthroplasties: a randomized controlled study. J Bone Joint Surg Am. 2010;92:2634-42.

15. McCalden RW, MacDonald SJ, Bourne RB, Marr JT. A randomized controlled trial comparing "high-flex" vs "standard" posterior cruciate substituting polyethylene tibial inserts in total knee arthroplasty. J Arthroplasty. 2009;24:338.

16. Choi $\mathrm{CH}$, Koo MH, Park YW. Comparative study of the postoperative maximal flexion angle in PCL-substituting TKAs: conventional PS vs. high-flex PS. J Korean Orthop Assoc. 2009;44:581-5.

17. Hirsch HS, Lotke PA, Morrison LD. The posterior cruciate ligament in total knee surgery. Save, sacrifice, or substitute? Clin Orthop Relat Res. 1994;(309):64-8.

18. Yoshiya S, Matsui N, Komistek RD, Dennis DA, Mahfouz M, Kurosaka M. In vivo kinematic comparison of posterior cruciate-retaining and posterior stabilized total knee arthroplasties under passive and weight-bearing conditions. J Arthroplasty. 2005;20:777-83.

19. Tanzer M, Smith K, Burnett S. Posterior-stabilized versus cruciate-retaining total knee arthroplasty: balancing the gap. J Arthroplasty. 2002;17:813-9.

20. Chaudhary R, Beaupre LA, Johnston DW. Knee range of motion during the first two years after use of posterior cruciate-stabilizing or posterior cruciate-retaining total knee prostheses. A randomized clinical trial. J Bone Joint Surg Am. 2008;90:2579-86.

21. Bertin KC. Cruciate-retaining total knee arthroplasty at 5 to 7 years followup. Clin Orthop Relat Res. 2005;(436):177-83.

22. Nerhus TK, Heir S, Thornes E, Madsen JE, Ekeland A. Time- 
dependent improvement in functional outcome following LCS rotating platform knee replacement. Acta Orthop. 2010;81:727-32.

23. Bin SI, Nam TS. Early results of high-flex total knee arthroplasty: comparison study at 1 year after surgery. Knee Surg Sports Traumatol Arthrosc. 2007;15:350-5.

24. Seng C, Yeo SJ, Wee JL, Subanesh S, Chong HC, Lo NN. Improved clinical outcomes after high-flexion total knee arthroplasty: a 5-year follow-up study. J Arthroplasty. 2011;26:1025-30.

25. Bellemans J, Banks S, Victor J, Vandenneucker H, Moemans A. Fluoroscopic analysis of the kinematics of deep flexion in total knee arthroplasty. Influence of posterior condylar offset. J Bone Joint Surg Br. 2002;84:50-3.

26. Bellemans J, Robijns F, Duerinckx J, Banks S, Vandenneucker
H. The influence of tibial slope on maximal flexion after total knee arthroplasty. Knee Surg Sports Traumatol Arthrosc. 2005;13:193-6.

27. Sierra RJ, Berry DJ. Surgical technique differences between posterior-substituting and cruciate-retaining total knee arthroplasty. J Arthroplasty. 2008;23:20-3.

28. Abbas D, Gunn RS. Medium-term results of the Scorpio total knee replacement. Knee. 2006;13:307-11.

29. Borrione F, Bonnevialle P, Mabit C, Guingand O, Bertin D, Bonnomet F, Denis C, Gagna G. Scorpio single radius total knee arthroplasty. A minimal five-year follow-up multicentric study. Int Orthop. 2011;35:1777-82.

30. Rand JA, Trousdale RT, Ilstrup DM, Harmsen WS. Factors affecting the durability of primary total knee prostheses. J Bone Joint Surg Am. 2003;85:259-65. 